\title{
UAV Development for Pollutions Monitoring
}

\author{
Baraa M. Albaker, Ibtihaj Abdulwahhab, Malik A. Al-saedi, Riam A. Esmaeel, Hind A. Kadum
}

\begin{abstract}
Air Pollution, due to car emissions; chemicals; dust...etc., can cause a variety of problems to humans, animals, plants and water resources. The main idea of this paper is to develop a tool that measures and remotely monitors air-quality and air-pollution status at different altitudes using different kinds of pollution sensors. A quad-copter in X-configuration, equipped with sensors utilized for detecting air pollutions are developed and integrated. The work also involves building a convenient graphic user interface to remotely monitor and determine air pollution levels, and provide a way to save pollution data for further offline processing and analyses.
\end{abstract}

Index Terms - Air quality, Monitoring and detection, Pollution, Quad-copter, UAV

\section{INTRODUCTION}

Nowadays, Unmanned Aerial Vehicles (UAVs) are currently deployed in numerous applications, especially in dangerous and dull tasks. A UAV system comprises integration of several hardware subsystems including sensors, actuators, and controllers. It also comprises many software components, like autopilot control, trajectory generation, mission execution, image processing, etc. [1-5]. Low-cost, small and autonomous multi-copter were increasingly used in many application areas instead of expensive and large aircraft platforms due to the advancement in computation processing units and development of powerful and small sensors.

Air pollution, like chemicals that are generated from factories; car emissions; and dust, is a combination of gases and solid particles. Climate is directly changed by particulate pollutions through heat and light absorption by black materials and incoming solar radiation scattering [6-8]. Some particles are water soluble and result in altering the lifetime and brightness of clouds [9]. The combinations of these effects will result in a reducing reachable sunlight [10]. This in turn have an impact on wind speeds and generated solar power [11]. Air quality monitoring gives insight into potential climate changes at a specified area and atmospheric level. Nonetheless, the majority of air pollution monitoring tasks are carried out at surface level while neglecting potential readings of higher altitude atmospheric levels. Pollutions at higher layers may not similar to the surface. Therefore, it is being a necessity to get the whole picture of pollutions to analyse climate change of an area.

Baraa Munqith Albaker, Networks Engineering Department, Al-Iraqia University, Baghdad, Iraq

Ibtihaj Abdulwahhab, Computer Engineering Department, Al-Iraqia University, Baghdad, Iraq

Malik A. Al-saedi, Networks Engineering Department, Al-Iraqia University, Baghdad, Iraq

Riam A. Esmaeel, Computer Engineering Department, Al-Iraqia University, Baghdad, Iraq

Hind A. Kadum, Computer Engineering Department, Al-Iraqia University, Baghdad, Iraq
One way to remotely sense and monitor air pollutions at different atmospheric levels is to use aircraft. It is considered a powerful tool especially when large areas need to be covered at short time. Manned aircraft requires airbases and trained pilots among other issues like fuel, platform cost, etc. $[5,12]$. This makes UAVs more attractive to perform this kind of tasks. Quad-copter platforms, as compared to fixed-wing UAVs, have numerous key advantages including hovering ability, and aggressive trajectory resolution. The deployment of this kind of UAV platforms in pollution sampling and monitoring allow safe, repeated flights, and inexpensive sampling of pollutions at diverse atmospheric layers.

A vertical take-off landing UAV of a small quad-copter type is developed for the purpose because of its characteristics, hover ability and low altitudes fly, that makes it suitable for this kind of missions. The detailed development methodology is presented in next sections, based on choosing right navigation and control components with suitable air-pollution sensors and building a convenient graphic user interface to present monitored data on a remote ground station.

\section{WORK METHODOLOGY}

Several steps are followed for the development and integration of the quad-copter. The first step, after studying several types of UAVs models, was to select right components for the development process of the quad-copter. The appropriate parts include low-level controller that controls the UAV movement, UAV frame, motors, Electronic Speed Controllers (ESCs), brushless DC motors, propellers, and battery size and power that is carefully selected to overcome demanding power by whole UAV's aviation electronics.

Later, individual UAV parts are integrated and low-level UAV control program is downloaded to the controller. After that, the testing and calibration process are carried out to test the direction of propellers according to predefined direction and calibrate the equipment, which further includes calibration of ESCs, motors, and microcontroller. Several test was then conducted to test UAV fly according to instructed directions. After that, pollution sensors were installed on the quad-copter's arms. Finally, several measurement are acquired at different areas and atmospheric layers.

\section{QUAD-COPTER INTEGRATION STEPS}

The required parts to develop complete quad-copter system are microcontroller, four electronic speed control "ESCs", four motors each of $2450 \mathrm{KV}$, four Propellers and radio controller transmitter and receiver subsystems.

The integration steps started by connecting electronic speed control (ESCs) to the frame board, microcontroller, and the motors. The motors are then connected to quad-copter's frame. Next, $\mathrm{RC}$ receiver are connected to the microcontroller after complete programming the controller for quad-copters $\mathrm{x}$-configuration. Thrust is created by the four propellers, 
which are driven by the four motors. Tests were conducted at this step to insure that any two motors in same axis have to rotate in same direction (for instance, clockwise direction) and reverse to other two motors in the other axis (counter-clockwise direction).

\section{PROPOSED LAyout OF THE QUAD-COPTER}

The proposed system consists of a microcontroller that Controls by movement of the quad-copter. It is used to stabilize and balance the UAV because the controller contains gyro and accelerometer sensors. The microcontroller is connected with $\mathrm{RC}$ receiver, which receives guidance information received from $\mathrm{RC}$ transmitter and passes them to microcontroller and accordingly the controller changes movement of quad-copter by controlling the speed of motors that use ESCs. The function of ESCs is to control the speed of motors. Fig. 1 shows the basic layout overview of the developed quad-copter.

Different kinds of air quality and pollution sensors are used and integrated with on-board UAV's sensors. This is to sense levels of different kinds of air pollution parameters at different altitudes.

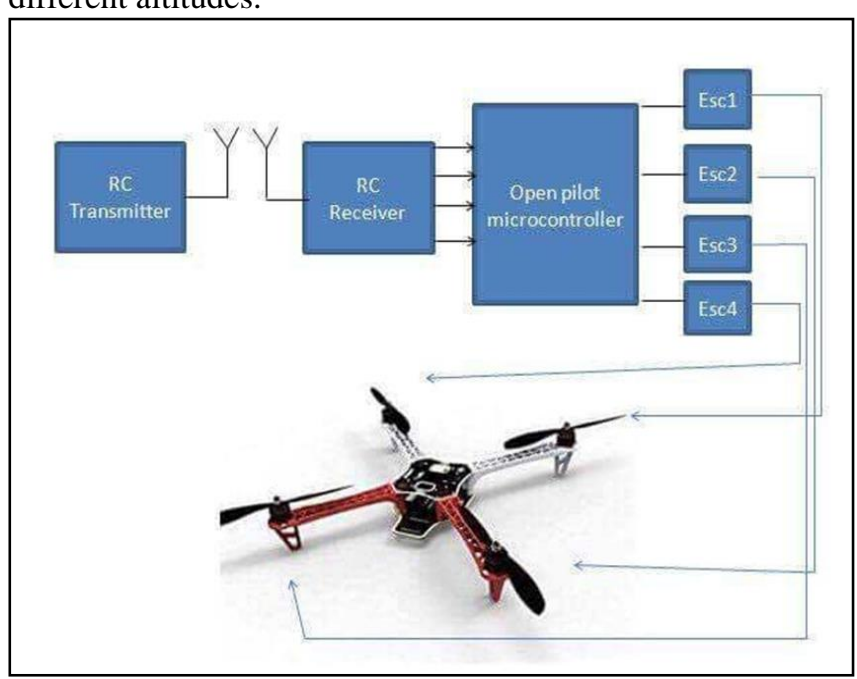

Fig. 1: Basic layout of the overall Quad-copter

A bidirectional Bluetooth communication link is developed to communicate with a ground station. The work also considers development of an effective operator interface. This is to further analyse received data, extract useful information and monitor air pollutions levels.

\section{QUAD-COPTER OPERATION AND CONTROL}

The RC Transmitter is used to control quad-copter movements in different modes and directions, involving up, down, left and right movement commands for the quad-copter. These movements can be achieved by changing the speed of motors.

In order to instruct UAV to fly to either left or right, a Yaw command is issued to rotate the head of the quad-copter. In this case, the autopilot controller spins two motors that are located in one line faster than two other motors in perpendicular line to generate enough thrust to rotate the $\mathrm{UAV}$ at the designated direction.

To control the forward and backward movement of the UAV, the autopilot controls the pitch angle according to commands set by a remote operator through the RC transmitter's aileron stick. When a forward command is issued, the autopilot force the quad-copter to tilt by slow-down the two propellers in front and speed-up the other two propellers in the back. Similarly, in case of backward movement, the autopilot speed-up the front propellers and slower-down the two in the back.

Finally, right and left manoeuvres of the quad-copter are controlled by the roll angle. Upon receiving left command from RC transmitter's aileron stick, the autopilot instruct UAV to fly right or left by increasing the thrust of two adjacent propellers while decreasing thrust of other two propellers.

\section{AIR POLLUTION SENSORS}

In this paper, we use different kinds of air quality and pollution sensors, including: MQ7,MQ6, MQ3, and MQ135 as presented in Fig. 2 below.

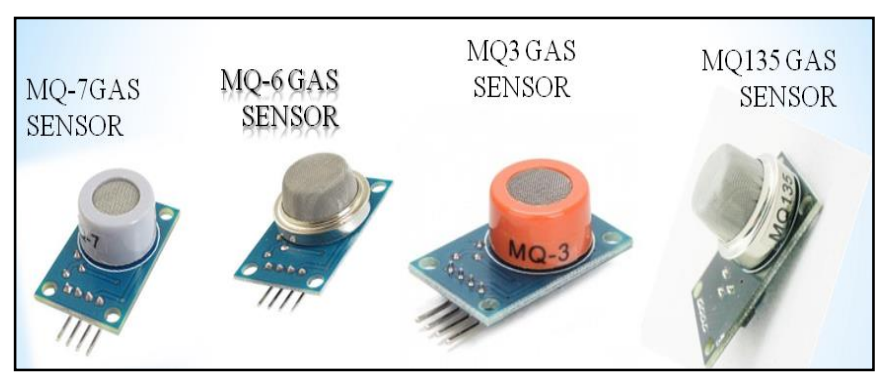

Fig. 2: Used types of air quality and pollution sensors

\section{QUAD-COPTER OPERATION AND CONTROL}

After completing the development and integration of quad-copter parts and calibration process for the microcontroller and the ESCs, we deployed it in different flying scenarios and monitored pollution levels at different atmospheric layers.

The results show better UAV performance and more accurately response to the control commands set by the RC. However, we faced other problems regarding synchronized movement of all propellers. Therefore, we decided to replace all ESCs and after testing the UAV, the results show correct response of the UAV according to desired commands issued by the operator. Fig. 3 shows the final version of the developed quad-copter with the set of MQ sensors.

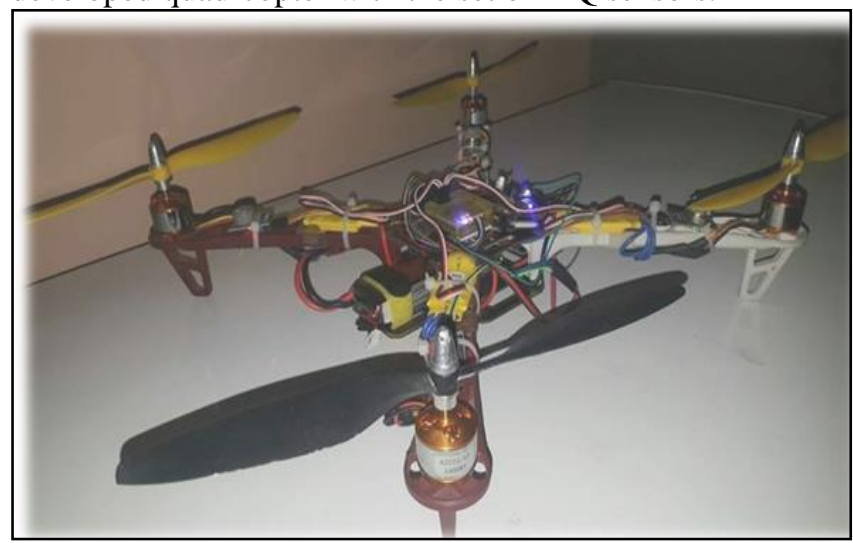

Fig. 3: Final version of the developed quad-copter with set of MQ sensors 
The system consists of a microcontroller, which is the heart of the multi-copter that controls the movement of the UAV. It is used to stabilize and balance the UAVs because the controller contains gyro and accelerometer sensors. The microcontroller is connected to a receiver, which receives the incoming routing information from the transmitter and passes it to the microcontroller accordingly. The control of the quadcopter movement changes by controlling the speed of the motors using ESCs. The ESCs were successfully calibrated to provide zero throttle for the propellers at no command, stable throttle value at hovering, and full throttle value to maximum motor applied current to rotate the propellers at maximum speed.

\section{DEVELOPED GUI FOR AIR POLLUTION MONITORING}

We developed a program in Visual Basic act as a graphical user interface (GUI) for a remote ground operator to display the results of the sensors attached on board of the UAV. Four different air-pollution sensors were used to sense pollution levels. The sensed data was transmitted via Bluetooth link to the ground station.

After testing in one of the nearby areas, we got results on air pollution. These results were transferred to the $\mathrm{PC}$ via the Bluetooth link connected to the UAV. Fig. 4 shows the developed GUI with a sampled air-pollution data. The received data can be displayed and saved in an Excel document format. This allows to off-line analysis to the data for further extracting useful information.

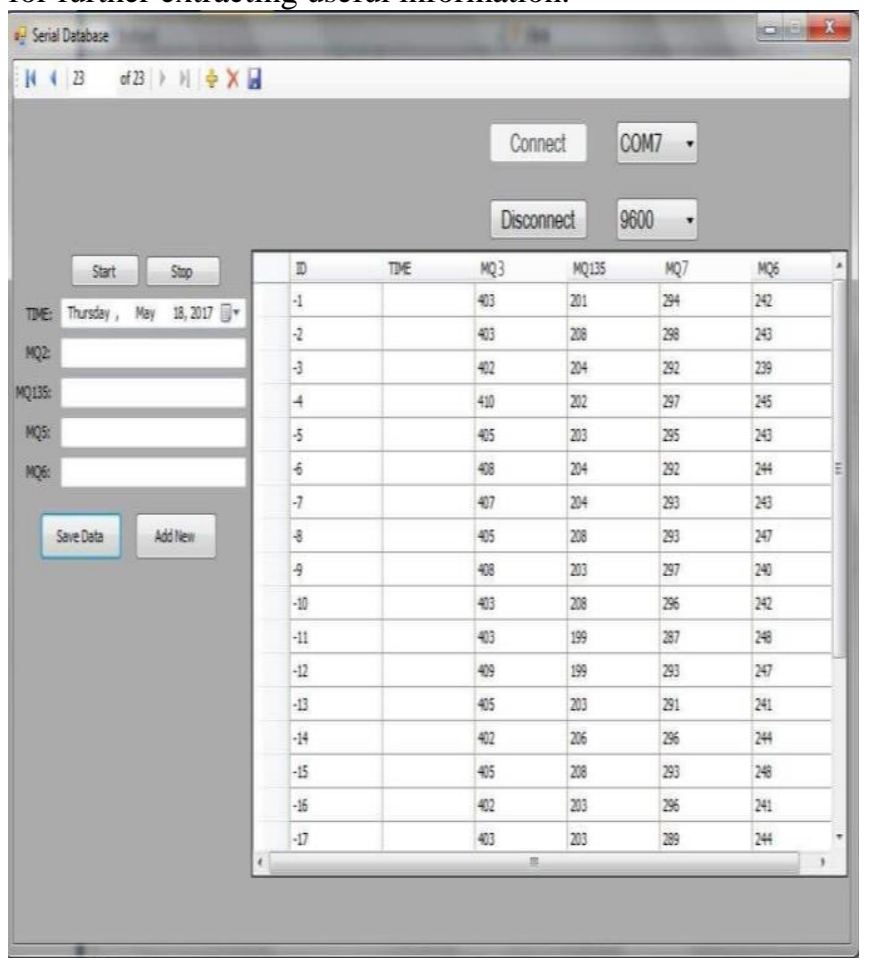

Fig. 4: Development VB.net graphic user interface.

\section{CONCLUSIONS}

UAV can be used as a new platform for remote sensing of chemicals in the environment at different altitudes, to provide wide-range air-pollution information monitoring tool. In this paper, a convenient mobile tool to measure and remotely monitor air-pollution levels at different altitudes was developed. Therefore, distribution of air-pollution levels can be extracted for a specific region. A quad-copter in
$\mathrm{X}$-configuration, equipped with sensors utilized for detecting air pollutions are developed and integrated. A convenient graphic user interface was also developed to remotely monitor and determine air pollution levels, and provide a way to save pollution data for further offline processing and analyses. Results show the successful implementation of the quad-copter to fly according to set commands. The results also show the successful implementation of GUI to read acquired on-board air-pollution data and display it in a convenient way to the ground operator.

\section{REFERENCES}

[1] H. Liu, Y. Bai, G. Lu, Z. Shi, and Y. Zhong, "Robust Tracking Control of a Quadrotor Helicopter," J. Intell. Robot. Syst., vol. 75, no. 3-4, 2014, pp. 595-608.

[2] B. M. Albaker and N. A. Rahim, "Flight Path PID Controller for Propeller-Driven Fixed-Wing Unmanned Aerial Vehicles," International Journal of Physical Sciences, Vol. 6, No.8, April 2011, pp. 1947-1964.

[3] Y. Bi and H. Duan, "Implementation of autonomous visual tracking and landing for a low-cost quadrotor," Opt. - Int. J. Light Electron Opt., vol. 124, no. 18, 2013, pp. 3296-3300.

[4] B. M. Albaker, "Preliminary Architectonic Design for a Smart Solar-Powered UAV," IEEE Conference on Clean Energy and Technology (CEAT 2013), Bayview Hotel, Langkawi, Malaysia, 2013 , pp. 238-242.

[5] B. M. Albaker, N. A. Rahim and A. H. Rasheed, "Development of a Flight Simulation Test-Bed for a General Propeller-Driven Unmanned Aircraft," Journal of Applied Mechanics and Materials, Vol. 724, Jan. 2015, pp. 303-306

[6] Charlson, R.J., S.E. Schwartz, J.M. Hales, R.D. Cess, J.A. Coakley, J.E. Hansen, and D.J. Hofmann, Climate forcing by anthropogenic aerosols, Science, Vol. 255, 1992, pp. 423-430.

[7] Ramanathan, V., P. Crutzen, J. Lelieveld, A. Mitra, et. Al., Indian Ocean Experiment: An integrated analysis of the climate forcing and effects of the great Indo-Asian haze, J. Geophys. Res. , 2001, 28371-28398.

[8] Twomey, S., The influence of pollution on the short-wave albedo of clouds, J. Atmos. Sci., 34, 1977, 11491152.

[9] Corrigan, CE. and Novakov, T. Cloud condensation nucleus activity of organic compounds: a laboratory study. ATMOSPHERIC ENVIRONMENT, Vol. 33, no.17, 1999, pp. 2661-2668.

[10] Ramanathan, V., C. Chung, D. Kim, T. Bettge, L. Buja, J. T. Kiehl, W. M. Washington, Q. Fu, D. R. Sikka, and M. Wild. Inaugural Article: Atmospheric brown clouds: Impacts on South Asian climate and hydrological cycle. PNAS, Vol. 102, 2005, pp. 5326-5333.

[11] Jacobson, M. Z., and Y. J. Kaufman, Wind reduction by aerosol particles, Geophys. Res. Lett.,33, L24814, doi:10.1029/2006GL027838, 2006.

[12] Y. M. Zhang, a. Chamseddine, C. a. Rabbath, B. W. Gordon, C.-Y. Su, S. Rakheja, C. Fulford, J. Apkarian, and P. Gosselin, "Development of advanced FDD and FTC techniques with application to an unmanned quadrotor helicopter testbed," J. Franklin Inst., vol. 350, no. 9, 2013 , pp. 2396-2422. 